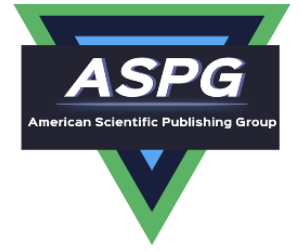

\title{
Introduction to Neutrosophic Subtraction Algebra and Neutrosophic Subtraction Semigroup
}

\author{
${ }^{1}$ M.A. Ibrahim, ${ }^{2}$ A.A.A. Agboola , ${ }^{3}$ E.O. Adeleke, ${ }^{4}$ S.A. Akinleye \\ $1,2,3,4$ Department of Mathematics, Federal University of Agriculture, Abeokuta, Nigeria. \\ muritalaibrahim40@gmail.com ${ }^{1}$,agboolaaaa@funaab.edu.ng ${ }^{2}$, yemi376@yahoo.com $^{3}$, \\ sa_akinleye@yahoo.com ${ }^{4}$
}

\begin{abstract}
The objective of this paper is to introduce and study the notion of neutrosophic subtraction algebras and neutrosophic subtraction semigroups. It also introduces the notion of neutrosophic ideals of neutrosophic subtraction semigroup and presents some of their basic properties. In addition, the notion of neutrosophic homomorphism of neutrosophic subtraction semigroups and neutrosophic quotient subtraction semigroups was also introduced.
\end{abstract}

Keywords: Neutrosophy, neutrosophic subtraction algebra,neutrosophic subtraction semigroup, neutrosophic Ideal.

\section{Introduction}

B. M. Schein studied in ${ }^{14}$ systems of the form $(\Phi, \circ, \backslash)$, where $\Phi$ is a set of functions closed under the composition " $\circ$ " of functions (and hence $(\Phi, \circ)$ is a function semi group), and closed under set theoretic subtraction " $\backslash$ " (and hence $(\Phi, \backslash)$ is a subtraction algebra. He proved that every subtraction semigroup is isomorphic to a difference semigroup of invertible functions. B. Zelinka ${ }^{27}$ discussed a problem proposed by B. M. Schein concerning the structure of multiplication in a subtraction semigroup. He solved the problem for subtraction algebras of a special type, called atomic subtraction algebras. Y. B. Jun, H.S. Kim and E. H. Roh ${ }^{5}$ introduced the notion of ideals in subtraction algebras and discussed the characterization of such ideals. In , 6 Y. B. Jun and H. S. Kim established the ideal generated by a set, and discussed related results. K.J. Lee, Y. B. Jun, and Y. H. Kim also introduced in,, 9 the notion of weak subtraction algebras and provided a method to make a weak subtraction algebra from a quasi-ordered set.

Neutrosophy is a new branch of philosophy that studies the origin, nature, and scope of neutralities, as well as their interactions with different ideational spectra. Neutrosophic set and neutrosophic logic were introduced in 1995 by Smarandache as generalizations of fuzzy set and respectively intuitionistic fuzzy logic. In neutrosophic logic, each proposition has a degree of truth $(T)$, a degree of indeterminancy $(I)$, and a degree of falsity $(F)$, where $T, I, F$ are standard or non-standard subsets of $]^{-} 0,1^{+}[$, see $15 \mid 16 ! 17$

The notion of neutrosophic algebraic structures was introduced by Kandasamy and Smarandache in 2006, see 24.25 Since then, several researchers have studied the concepts and a great deal of literature has been produced. For example, Agboola and Davvaz introduced the concept of neutrosophic BCI/BCK-algebras in.1 A comprehensive review of neutrosophy, neutrosophic triplet set and neutrosophic algebraic structures can be found in [3||1|1|11|12|13|18|19|20|21|22|23] . The present paper is concerned with introducing the concept of neutrosophic subtraction algebras and subtraction semigroup. Some of their elementary properties are presented.

\section{Preliminaries}

In this section, some basic definitions and properties that will be useful in this work are given. 
Definition 2.1. ${ }^{2}$ A pair $(A,-)$ where $A$ is a nonempty set and ' - ' is a binary operation on $\mathrm{A}$ is called a subtraction algebra if

1. $x-(y-x)=x$;

2. $x-(x-y)=y-(y-x)$ and

3. $(x-y)-z=(x-z)-y$ for all $x, y, z \in A$.

Axiom 3 of 2.1 permits us to omit parentheses in expressions of the form $(x-y)-z$. The subtraction determines an order relation on $A: x \leq y$ if and only if $x-y=0$, where $0=x-x$ is an element that does not depend on the choice of $x \in A$. The ordered set $(A, \leq)$ is a semi-Boolean algebra, that is, it is a meet semilattice with zero 0 in which every interval $[0, x]$ is a Boolean algebra with respect to the induced order. ${ }^{7}$ Here $x \wedge y=x-(x-y)$; the complement of an element $y \in[0, x]$ is $x-y$; and if $y, z \in[0, x]$, then

$$
y \vee z=\left(y^{\prime} \wedge z^{\prime}\right)^{\prime}=x-((x-y) \wedge(x-z))=x-((x-y)-((x-y)-(x-z))) .
$$

In a subtraction algebra, the following hold 7

1. $x-0=x$ and $0-x=0$

2. $x-(x-y) \leq y$.

3. $x \leq y$ if and only if $x=y-w$ for some $w \in X$.

4. $x \leq y$ implies $x-z \leq y-z$ and $z-y \leq z-x$ for all $z \in X$.

5. $x-(x-(x-y))=x-y$.

Definition 2.2. ${ }^{26}$ Let $X$ be a subtraction algebra and $Y$ a nonempty subset of $X$. Then $Y$ is called a subalgebra of $X$ if $x-y \in Y$ whenever $x, y \in Y$

Definition 2.3. ${ }^{2}$ A triple $(A,-, \cdot)$ is called a subtraction semigroup if

1. $(A,-)$ is a subtraction algebra;

2. $(A, \cdot)$ is a semigroup and

3. $x(y-z)=x y-x z$ and $(y-z) x=y x-z x$ for all $x, y, z \in A$.

A subtraction semigroup is said to be multiplicatively abelian if multiplication is commutative.

Definition 2.4. A triple $(A,-, \cdot)$ is called a subtraction group if

1. $(A,-, \cdot)$ is a subtraction semigroup and

2. $A-\{0\}$ is a group with the multiplication inherited from $A$.

Example 2.5. ${ }^{8}$ Let $X=\{0,1\}$ be a set in which " - " and " . " are defined by

Table 1: (a) Cayley table for the binary operation " - " and (b) Cayley table for the binary operation ". ."

\begin{tabular}{lll}
\hline- & 0 & 1 \\
\hline 0 & 0 & 0 \\
1 & 1 & 0 \\
\hline
\end{tabular}

(a)

\begin{tabular}{lll}
\hline$\cdot$ & 0 & 1 \\
\hline 0 & 0 & 0 \\
1 & 0 & 1 \\
\hline
\end{tabular}

(b)

It is easy to check that $\mathrm{X}$ is a subtraction semigroup.

Definition 2.6. A non empty set $X$ together with two binary operations ${ }^{\prime}{ }^{\prime}$ and ${ }^{\prime} \cdot$ is said to be a left near subtraction semigroup if

1. $(X,-)$ is a subtraction algebra

2. $(X, \cdot)$ is a semigroup and 
3. $z(x-y)=z x-z y$ for every $x, y, z \in X$.

Definition 2.7. A non empty set $X$ together with two binary operations ${ }^{\prime}{ }^{\prime}$ and ${ }^{\prime} \cdot$ is said to be a right near subtraction semigroup if

1. $(X,-)$ is a subtraction algebra

2. $(X, \cdot)$ is a semigroup and

3. $(x-y) z=x z-y z$ for every $x, y, z \in X$.

\section{Formulation of a Neutrosophic Subtraction Algebra and Neutrosophic Subtraction Semigroup}

In this section, the concept of neutrosophic subtraction algebra and subtraction semigroup are develop. Some of their basic properties are presented.

Definition 3.1. Let $X$ be a nonempty set. A set $X(I)=<X \cup I>$ generated by $X$ and $I$ is called a neutrosophic set. The elements of $X(I)$ are of the form $(x, y I)$ where $x$ and $y$ are elements of $X$. $I$ is called an indeterminate and it has the property $I^{n}=I$ for all positive integer $\mathrm{n}$.

Definition 3.2. Let $(X,-)$ be any classical subtraction algebra and let $X(I)=\langle X \cup I\rangle$ be a set generated by $X$ and $I$. Consider the neutrosophic algebraic structure $\left(X(I),{ }_{N}\right)$ where for all $(a, b I),(c, d I) \in X(I)$, $-N$ is defined by

$$
(a, b I)-{ }_{N}(c, d I)=(a-c,(b-d) I) \quad \forall a, b, c, d \in X
$$

We call $\left(X(I),-_{N}\right)$ a neutrosophic subtraction algebra.

An element $x \in X$ is represented by $(x, 0) \in X(I)$ and $(0,0)$ represents the constant element in $X(I)$.

Example 3.3. Let $X(I)=\{(0,0),(a, 0),(b, 0),(1,0),(0, a I),(0, b I),(0, I)\}$ be a neutrosophic set in which " ${ }_{N}$ " is defined as in the table below.

Table 2: Cayley table for the binary operation $-{ }_{N}$

\begin{tabular}{llllllll}
\hline$-_{N}$ & $(0,0)$ & $(a, 0)$ & $(b, 0)$ & $(1,0)$ & $(0, a I)$ & $(0, b I)$ & $(0, I)$ \\
\hline$(0,0)$ & $(0,0)$ & $(0,0)$ & $(0,0)$ & $(0,0)$ & $(0,0)$ & $(0,0)$ & $(0,0)$ \\
$(a, 0)$ & $(a, 0)$ & $(0,0)$ & $(a, 0)$ & $(0,0)$ & $(a, 0)$ & $(a, 0)$ & $(a, 0)$ \\
$(b, 0)$ & $(b, 0)$ & $(b, 0)$ & $(0,0)$ & $(0,0)$ & $(b, 0)$ & $(b, 0)$ & $(b, 0)$ \\
$(1,0)$ & $(1,0)$ & $(b, 0)$ & $(a, 0)$ & $(0,0)$ & $(1,0)$ & $(1,0)$ & $(1,0)$ \\
$(0, a I)$ & $(0, a I)$ & $(0, a I)$ & $(0, a I)$ & $(0, a I)$ & $(0,0)$ & $(0, a I)$ & $(0,0)$ \\
$(0, b I)$ & $(0, b I)$ & $(0, b I)$ & $(0, b I)$ & $(0, b I)$ & $(0, b I)$ & $(0,0)$ & $(0,0)$ \\
$(0, I)$ & $(0, I)$ & $(0, I)$ & $(0, I)$ & $(0, I)$ & $(0, b I)$ & $(0, a I)$ & $(0,0)$ \\
\hline
\end{tabular}

Then $\left(X(I),-_{N}\right)$ is a neutrosophic subtraction algebra.

Proposition 3.4. Every neutrosophic subtraction algebra $\left(X(I),{ }_{N}\right)$ is a subtraction algebra.

Proof: Suppose that $\left(X(I),{ }_{N}\right)$ is a subtraction algebra.

Let $x=(a, b I), y=(c, d I), z=(e, f I) \in X(I)$ with $a, b, c, d, e, f \in X$.

Then

1. We show that $x-{ }_{N}\left(y-{ }_{N} x\right)=x$

$$
\begin{aligned}
x-{ }_{N}\left(y-{ }_{N} x\right) & =(a, b I)-{ }_{N}\left((c, d I)-{ }_{N}(a, b I)\right) \\
& =(a, b I)-{ }_{N}(c-a,(d-b) I) \\
& =(a-(c-a),(b-(d-b)) I) \\
& =(a, b I) \quad \text { Since a,b,c,d } \in X \\
& =x .
\end{aligned}
$$


2. We want to show that $x-{ }_{N}\left(x-{ }_{N} y\right)=y-{ }_{N}\left(y-{ }_{N} x\right)$

$$
\begin{array}{rlr}
x-{ }_{N}\left(x-{ }_{N} y\right) & =(a, b I)-{ }_{N}((a, b I)-N(c, d I)) \\
& =(a, b I)-{ }_{N}((a-c),(b-d) I) \\
& =(a-(a-c),(b-(b-d))) I \\
& =(c-(c-a),(d-(d-b))) I \quad \text { Since a,b,c, d } \in X \\
& =(c, d I)-{ }_{N}((c-a),(d-b) I) \\
& =(c, d I)-{ }_{N}((c, d I)-N(a, b I)) \\
& =y-{ }_{N}\left(y-{ }_{N} x\right) .
\end{array}
$$

3. We want to show that $\left(x-{ }_{N} y\right)-{ }_{N} z=\left(x-{ }_{N} z\right)-{ }_{N} y$

$$
\begin{aligned}
\left(x-{ }_{N} y\right)--_{N} z & =((a, b I)-N(c, d I))-{ }_{N}(e, f I) \\
& =(a-c,(b-d) I)-N(e, f I) \\
& =(a-c)-e,((b-d)-f) I \\
& =(a-e)-c,((b-f)-d) I \quad \text { Since a,b,c,d,e,f } \in X \\
& =((a-e),(b-f) I)-{ }_{N}(c, d I) \\
& =\left((a, b I)-{ }_{N}(e, f I)\right)-{ }_{N}(c, d I) \\
& =(x-N)-N y .
\end{aligned}
$$

$\forall x, y, z \in X(I)$

From ${ }^{24}$ we note that, if $x \leq y$ then we can not in general say $x I \leq y I$, it may so happen that $y I \leq x I$. Thus the neutrosophic order in general need not to preserve the order. If a set $X$ is ordered under ${ }^{\prime} \leq^{\prime}$ then the neutrosophic part of $\langle X \cup I>$ may or may not have the preservations of order under $\leq$; i.e., if $x \leq y$, $x, y \in X$ then $x I \leq y I$ may occur or may not occur. For the purpose of this work suppose $x I \leq y I$ occur.

Proposition 3.5. If $\left(X(I),{ }_{N}\right)$ is a neutrosophic subtraction algebra, then the relation $\leq$ is a partial order on $X(I)$.

Proof: Let $(a, b I),(c, d I),(e, f I) \in X(I)$ and $a, b, c, d, e, f \in X$.

1. Since $(a, b I)-{ }_{N}(a, b I)=(a-a,(b-b) I)=(0,0 I)$ then $(a, b I) \leq(a, b I)$

2. Suppose that $(a, b I) \leq(c, d I)$ and $(c, d I) \leq(a, b I)$

then $(a, b I)-{ }_{N}(c, d I)=(0,0 I)$ implies $(a-c,(b-d) I)=(0,0 I)$

and $(c, d I)-{ }_{N}(a, b I)=(0,0 I)$ implies $(c-a,(d-b) I)=(0,0 I)$.

Now,

$$
\begin{aligned}
& (a, b I)=(a, b I)-{ }_{N}(0,0 I) \\
& =(a-0,(b-0) I) \\
& =(a-(a-c),(b-(b-d)) I) \quad \text { Since, } \mathrm{a}-\mathrm{c}=0 \text { and } \mathrm{b}-\mathrm{d}=0 \\
& =(c-0,(d-0) I) \\
& =(c, d I)
\end{aligned}
$$$$
=(c-(c-a),(d-(d-b)) I) \quad \text { Since } \mathrm{X} \text { is a subtraction algebra }
$$

Since, $c-a=0$ and $d-b=0$

3. Suppose that $(a, b I) \leq(c, d I)$ and $(c, d I) \leq(e, f I)$

$$
\begin{aligned}
& \text { then }(a, b I)-{ }_{N}(c, d I)=(0,0 I) \text { implies }(a-c,(b-d) I)=(0,0 I) \\
& \begin{aligned}
\text { and }(c, d I)-{ }_{N}(e, f I) & =(0,0 I) \text { implies }(c-e,(d-f) I)=(0,0 I) . \\
(a, b I)-N(e, f I) & =(a-e,(b-f) I) \\
& =((a-e)-0,((b-f)-0) I) \\
& =((a-e)-(a-c),((b-f)-(b-d)) I) \\
& =((a-(a-c))-e,((b-(b-d))-f) I) \\
& =((c-(c-a))-e,((d-(d-b))-f) I) \\
& =((c-e)-(c-a),((d-f)-(d-b)) I) \\
& =(0-(c-a),(0-(d-b)) I) \\
& =(0,0 I)
\end{aligned}
\end{aligned}
$$

Hence $(a, b I) \leq(e, f I)$. Consequently $\leq$ is a partial order.

In the next proposition some important properties of neutrosophic subtraction algebra which will be frequently used throughout this work are provided with their proofs.

Proposition 3.6. Let $\left(X(I),-_{N}\right)$ be a neutrosophic subtraction algebra. If $(a, b I),(c, d I) \in X(I)$, with $a, b, c, d \in X$ then the following are true.

$$
\text { 1. }(a, b I)-{ }_{N}(0,0 I)=(a, b I)
$$


2. $(0,0 I)-{ }_{N}(a, b I)=(0,0 I)$

3. $\left((a, b I)-{ }_{N}(c, d I)\right)-{ }_{N}(a, b I)=(0,0 I)$

4. $\left((a, b I)-{ }_{N}(c, d I)\right)-{ }_{N}(c, d I)=(a, b I)-{ }_{N}(c, d I)$

5. $\left((a, b I)-{ }_{N}(c, d I)\right)-{ }_{N}\left((c, d I)-{ }_{N}(a, b I)\right)=(a, b I)-{ }_{N}(c, d I)$

6. $(a, b I)-{ }_{N}\left((a, b I)-{ }_{N}\left((a, b I)-{ }_{N}(c, d I)\right)\right)=(a, b I)-{ }_{N}(c, d I)$

7. $(a, b I)-{ }_{N}\left((a, b I)-{ }_{N}(c, d I)\right) \leq(c, d I)$

8. $(a, b I)=(c, d I)$ iff $(a, b I)-{ }_{N}(c, d I)=(0,0 I)$ and $(c, d I)=(a, b I)$

Proof:

1. $(a, b I)-{ }_{N}(0,0 I)=(a-0,(b-0) I)=(a-(a-a),(b-(b-b)) I)=(a, b I)$.

2. $(0,0 I)-{ }_{N}(a, b I)=(0-a,(0-b) I)=(0-(a-0),(0-(b-0)) I)=(0,0 I)$.

$$
\begin{aligned}
3 .\left((a, b I)-{ }_{N}(c, d I)\right)-{ }_{N}(a, b I) & =(a-c,(b-d) I)-{ }_{N}(a, b I) \\
& =((a-c)-a,((b-d)-b) I) \\
& =((a-a)-c,((b-b)-d) I) \\
& =(0-c,(0-d) I) \\
& =(0,0 I) . \\
4 .\left((a, b I)-{ }_{N}(c, d I)\right)-{ }_{N}(c, d I) & =(a-c,(b-d) I)-{ }_{N}(c, d I) \\
& =((a-c)-c,((b-d)-d) I) \\
& =((a-c)-(c-(a-c)),((b-d)-(d-(b-d)) I) \\
& =((a-c),(b-d) I) \\
& =(a, b I)-{ }_{N}(c, d I) .
\end{aligned}
$$

$$
\begin{aligned}
5 .\left((a, b I)-{ }_{N}(c, d I)\right)-{ }_{N}\left((c, d I)-{ }_{N}(a, b I)\right) & =(a-c,(b-d) I)-{ }_{N}(c-a,(d-b) I) \\
& =((a-c)-(c-a),((b-d)-(d-b)) I) \\
& =((a-(c-a)-c,((b-(d-b))-d) I) \\
& =(a-c,(b-d) I) \\
& =(a, b I)-{ }_{N}(c, d I) . \\
6 .(a, b I)-{ }_{N}\left((a, b I)-{ }_{N}\left((a, b I)-{ }_{N}(c, d I)\right)\right) & =(a, b I)-{ }_{N}((a, b I)-N(a-c,(b-d) I)) \\
& =(a, b I)-N(a-(a-c),(b-(b-d)) I) \\
& =(a-(a-(a-c)),(b-(b-(b-d))) I) \\
& =((a-c)-((a-c)-a),((b-d)-((b-d)-b)) I)
\end{aligned}
$$

Since from the properties of $\mathrm{X}$, if $a, c \in X$ then $(a-c)-a=0$ then we have

$$
\begin{aligned}
((a-c)-((a-c)-a),((b-d)-((b-d)-b)) I) & =((a-c)-0,((b-d)-0) I) \\
& =(a-c,(b-d) I) \\
& =(a, b I)-{ }_{N}(c, d I) .
\end{aligned}
$$

7. $\left((a, b I)-{ }_{N}\left((a, b I)-{ }_{N}(c, d I)\right)\right)-{ }_{N}(c, d I)=\left((a, b I)-{ }_{N}(c, d I)\right)-{ }_{N}\left((a, b I)-{ }_{N}(c, d I)\right)=(0,0 I)$ $\Longrightarrow(a, b I)-{ }_{N}\left((a, b I)-{ }_{N}(c, d I)\right) \leq(c, d I)$.

8. Suppose that $(a, b I)-{ }_{N}(c, d I)=(0,0 I)$ and $(c, d I)-{ }_{N}(a, b I)=(0,0 I)$. Then

$$
\begin{aligned}
(a, b I) & =(a, b I)-{ }_{N}(0,0 I) \\
& =(a, b I)-{ }_{N}\left((a, b I)-_{N}(c, d I)\right) \\
& =(c, d I)-{ }_{N}\left((c, d I)-{ }_{N}(a, b I)\right) \\
& =(c, d I)-{ }_{N}(0,0 I) \\
& =(c, d I) . \\
& \Longrightarrow(a, b I)=(c, d I) .
\end{aligned}
$$

Conversely, suppose that $(a, b I)=(c, d I)$

Then $(a, b I)-{ }_{N}(c, d I)=(a, b I)-{ }_{N}(a, b I)=(0,0 I)$ and $(c, d I)-{ }_{N}(a, b I)=(c, d I)-{ }_{N}(c, d I)=(0,0 I)$ $\Longrightarrow(a, b I)-{ }_{N}(c, d I)=(0,0 I)$ and $(c, d I)-{ }_{N}(a, b I)=(0,0 I)$. 
Definition 3.7. Let $X_{1}(I)$ and $X_{2}(I)$ be two neutrosophic subtraction algebra. The direct product of $X_{1}(I)$ and $X_{2}(I)$ denoted by $X_{1}(I) \times X_{2}(I)$ is defined by

$X_{1}(I) \times X_{2}(I)=\left\{\left(\left(a_{1}, b_{1} I\right),\left(a_{2}, b_{2} I\right)\right):\left(a_{1}, b_{1} I\right) \in X_{1}(I),\left(a_{2}, b_{2} I\right) \in X_{2}(I)\right\}$.

Proposition 3.8. Let $\left(X_{1}(I),-N_{1}\right)$ and $\left(X_{2}(I),-N_{2}\right)$ be two neutrosophic subtraction algebra then $\left(X_{1}(I) \times X_{2}(I),-_{N}\right)$ is a neutrosophic subtraction algebra.

Proof:

Let $x=\left(\left(a_{1}, b_{1} I\right),\left(a_{2}, b_{2} I\right)\right), y=\left(\left(c_{1}, d_{1} I\right),\left(c_{2}, d_{2} I\right)\right), z=\left(\left(e_{1}, f_{1} I\right),\left(e_{2}, f_{2} I\right)\right) \in X_{1}(I) \times X_{2}(I)$ for all $a_{1}, b_{1}, c_{1}, d_{1} \in X_{1}$ and $a_{2}, b_{2}, c_{2}, d_{2} \in X_{2}$ then

1. we shall show that $x-{ }_{N}\left(y-{ }_{N} x\right)=x$

$$
\begin{aligned}
x-{ }_{N}\left(y-{ }_{N} x\right) & =\left(\left(a_{1}, b_{1} I\right),\left(a_{2}, b_{2} I\right)\right)-{ }_{N}\left[\left(\left(c_{1}, d_{1} I\right),\left(c_{2}, d_{2} I\right)\right)-{ }_{N}\left(\left(a_{1}, b_{1} I\right),\left(a_{2}, b_{2} I\right)\right)\right] \\
& =\left(\left(a_{1}, b_{1} I\right),\left(a_{2}, b_{2} I\right)\right)-N_{N}\left[\left(c_{1}, d_{1} I\right)-N_{1}\left(a_{1}, b_{1} I\right),\left(c_{2}, d_{2} I\right)-N_{2}\left(a_{2}, b_{2} I\right)\right] \\
& =\left(a_{1}, b_{1} I\right)-N_{1}\left(\left(c_{1}, d_{1} I\right)-N_{1}\left(a_{1}, b_{1} I\right)\right),\left(a_{2}, b_{2}\right)-N_{2}\left(\left(c_{2}, d_{2} I\right)-N_{2}\left(a_{2}, b_{2} I\right)\right) \\
& =\left(\left(a_{1}, b_{1} I\right),\left(a_{2}, b_{2} I\right)\right) \\
& =x .
\end{aligned}
$$

2. We want to show that $x-{ }_{N}\left(x-{ }_{N} y\right)=y-{ }_{N}\left(y-{ }_{N} x\right)$

$$
\begin{aligned}
x--_{N}(x-N y) & =\left(\left(a_{1}, b_{1} I\right),\left(a_{2}, b_{2} I\right)\right)--_{N}\left[\left(\left(a_{1}, b_{1} I\right),\left(a_{2}, b_{2} I\right)\right)-N_{N}\left(\left(c_{1}, d_{1} I\right),\left(c_{2}, d_{2} I\right)\right)\right] \\
& =\left(\left(a_{1}, b_{1} I\right),\left(a_{2}, b_{2} I\right)\right)-N_{N}\left[\left(\left(a_{1}, b_{1} I\right)-N_{1}\left(c_{1}, d_{1} I\right)\right),\left(\left(a_{2}, b_{2} I\right)-N_{2}\left(c_{2}, d_{2} I\right)\right)\right] \\
& =\left(a_{1}, b_{1} I\right)-N_{1}\left(\left(a_{1}, b_{1} I\right)-N_{1}\left(c_{1}, d_{1} I\right)\right),\left(a_{2}, b_{2} I\right)-N_{2}\left(\left(a_{2}, b_{2} I\right)-N_{2}\left(c_{2}, d_{2} I\right)\right) \\
& =\left(c_{1}, d_{1} I\right)-N_{1}\left(\left(c_{1}, d_{1} I\right)-N_{1}\left(a_{1}, b_{1} I\right)\right),\left(c_{2}, d_{2} I\right)-N_{2}\left(\left(c_{2}, d_{2} I\right)-N_{2}\left(a_{2}, b_{2} I\right)\right) \\
& =\left(\left(c_{1}, d_{1} I\right),\left(c_{2}, d_{2} I\right)-{ }_{N}\left[\left(\left(c_{1}, d_{1} I\right)-N_{1}\left(a_{1}, b_{1} I\right)\right),\left(\left(c_{2}, d_{2} I\right)-N_{2}\left(a_{2}, b_{2} I\right)\right)\right]\right. \\
& =\left(\left(c_{1}, d_{1} I\right),\left(c_{2}, d_{2} I\right)--_{N}\left[\left(\left(c_{1}, d_{1} I\right),\left(c_{2}, d_{2} I\right)\right)-{ }_{N}\left(\left(a_{1}, b_{1} I\right),\left(a_{2}, b_{2} I\right)\right)\right]\right. \\
& =y-N(x-N y) .
\end{aligned}
$$

3. We want to show that $\left(x-{ }_{N} y\right)-{ }_{N} z=\left(x-{ }_{N} z\right)-{ }_{N} y$

$$
\begin{aligned}
\left(x-{ }_{N} y\right)-{ }_{N} z & =\left[\left(\left(a_{1}, b_{1} I\right),\left(a_{2}, b_{2} I\right)\right)-{ }_{N}\left(\left(c_{1}, d_{1} I\right),\left(c_{2}, d_{2} I\right)\right)\right]-{ }_{N}\left(\left(e_{1}, f_{1} I\right),\left(e_{2}, f_{2} I\right)\right) \\
& =\left[\left(\left(a_{1}, b_{1} I\right)-N_{1}\left(c_{1}, d_{1} I\right)\right),\left(\left(a_{2}, b_{2} I\right)-N_{2}\left(c_{2}, d_{2} I\right)\right)\right]--_{N}\left(\left(e_{1}, f_{1} I\right),\left(e_{2}, f_{2} I\right)\right) \\
& =\left(\left(a_{1}, b_{1} I\right)-N_{1}\left(c_{1}, d_{1} I\right)\right)-N_{1}\left(e_{1}, f_{1} I\right),\left(\left(a_{2}, b_{2} I\right)-N_{2}\left(c_{2}, d_{2} I\right)\right)-N_{2}\left(e_{2}, f_{2} I\right) \\
& =\left(\left(a_{1}, b_{1} I\right)-N_{1}\left(e_{1}, f_{1} I\right)\right)-N_{1}\left(c_{1}, d_{1} I\right),\left(\left(a_{2}, b_{2} I\right)-N_{2}\left(e_{2}, f_{2} I\right)\right)-N_{2}\left(c_{2}, d_{2} I\right) \\
& =\left[\left(\left(a_{1}, b_{1} I\right)-N_{1}\left(e_{1}, f_{1} I\right)\right),\left(\left(a_{2}, b_{2} I\right)-N_{2}\left(e_{2}, f_{2} I\right)\right)\right]--_{N}\left(\left(c_{1}, d_{1} I\right),\left(c_{2}, d_{2} I\right)\right) \\
& =\left[\left(\left(a_{1}, b_{1} I\right),\left(a_{2}, b_{2} I\right)\right)-{ }_{N}\left(\left(e_{1}, f_{1} I\right),\left(e_{2}, f_{2} I\right)\right)\right]-{ }_{N}\left(\left(c_{1}, d_{1} I\right),\left(c_{2}, d_{2} I\right)\right) \\
& =(x-N z)-{ }_{N} y .
\end{aligned}
$$

Proposition 3.9. Let $X_{1}(I)$ be a neutrosophic subtraction algebra and let $A$ be a classical subtraction algebra then $\left(X_{1}(I) \times A,{ }_{N}\right)$ is a neutrosophic subtraction algebra.

Proof : It follows similar approach as in 3.8 above.

Definition 3.10. Let $\left(X(I),{ }_{N}\right)$ be a neutrosophic subtraction algebra. A nonempty subset $A(I)$ is called a neutrosophic subtraction subalgebra of $X(I)$ if the following conditions hold:

1. If $A(I) \neq \emptyset$

2. $(a, b I)-{ }_{N}(c, d I) \in A(I)$ for all $(a, b I),(c, d I) \in A(I)$.

3. $A(I)$ contains a proper subset which is a subtraction algebra.

If $A(I)$ does not contain a proper subset which is a subtraction algebra, then $A(I)$ is called a pseudo neutrosophic subtraction subalgebra of $X(I)$.

Example 3.11. Any neutrosophic subset of the neutrosophic set $X(I)$ of Example 3.3 is a neutrosophic subtraction subalgebra.

Definition 3.12. Let $(X,-, \cdot)$ be any subtraction semigroup and let $X(I)=\langle X \cup I\rangle$ be a set generated by $X$ and $I$. The triple $\left(X(I),-{ }_{N}, \cdot\right)$ is called a neutrosophic subtraction semi-group. If $(a, b I)$ and $(c, d I)$ are any two elements of $X(I)$ with $a, b, c, d \in X$, we define

$$
(a, b I)-{ }_{N}(c, d I)=(a-c,(b-d) I)
$$

and

$$
(a, b I) \cdot(c, d I)=(a c,(a d+b c+b d) I) .
$$


Example 3.13. Let $X(I)=\{(0,0),(a, 0),(b, 0),(1,0),(0, a I),(0, b I),(0, I)\}$ be a neutrosophic set in which $"-{ }_{N} "$ and "." are defined as in the tables below.

Table 3: (i) Cayley table for the binary operation " ${ }_{-}$" and (ii) Cayley table for the binary operation ". "

(i)

\begin{tabular}{llllllll}
\hline$-_{N}$ & $(0,0)$ & $(a, 0)$ & $(b, 0)$ & $(1,0)$ & $(0, a I)$ & $(0, b I)$ & $(0, I)$ \\
\hline$(0,0)$ & $(0,0)$ & $(0,0)$ & $(0,0)$ & $(0,0)$ & $(0,0)$ & $(0,0)$ & $(0,0)$ \\
$(a, 0)$ & $(a, 0)$ & $(0,0)$ & $(a, 0)$ & $(0,0)$ & $(a, 0)$ & $(a, 0)$ & $(a, 0)$ \\
$(b, 0)$ & $(b, 0)$ & $(b, 0)$ & $(0,0)$ & $(0,0)$ & $(b, 0)$ & $(b, 0)$ & $(b, 0)$ \\
$(1,0)$ & $(1,0)$ & $(b, 0)$ & $(a, 0)$ & $(0,0)$ & $(1,0)$ & $(1,0)$ & $(1,0)$ \\
$(0, a I)$ & $(0, a I)$ & $(0, a I)$ & $(0, a I)$ & $(0, a I)$ & $(0,0)$ & $(0, a I)$ & $(0,0)$ \\
$(0, b I)$ & $(0, b I)$ & $(0, b I)$ & $(0, b I)$ & $(0, b I)$ & $(0, b I)$ & $(0,0)$ & $(0,0)$ \\
$(0, I)$ & $(0, I)$ & $(0, I)$ & $(0, I)$ & $(0, I)$ & $(0, b I)$ & $(0, a I)$ & $(0,0)$ \\
\hline
\end{tabular}

(ii)

\begin{tabular}{llllllll}
\hline$\cdot$ & $(0,0)$ & $(a, 0)$ & $(b, 0)$ & $(1,0)$ & $(0, a I)$ & $(0, b I)$ & $(0, I)$ \\
\hline$(0,0)$ & $(0,0)$ & $(0,0)$ & $(0,0)$ & $(0,0)$ & $(0,0)$ & $(0,0)$ & $(0,0)$ \\
$(a, 0)$ & $(0,0)$ & $(a, 0)$ & $(0,0)$ & $(a, 0)$ & $(0, a I)$ & $(0,0)$ & $(0, a I)$ \\
$(b, 0)$ & $(0,0)$ & $(0,0)$ & $(b, 0)$ & $(b, 0)$ & $(0,0)$ & $(0, b I)$ & $(0, b I)$ \\
$(1,0)$ & $(0,0)$ & $(a, 0)$ & $(b, 0)$ & $(1,0)$ & $(0, a I)$ & $(0, b I)$ & $(0, I)$ \\
$(0, a I)$ & $(0,0)$ & $(0, a I)$ & $(0,0)$ & $(0, a I)$ & $(0, a I)$ & $(0,0)$ & $(0, a I)$ \\
$(0, b I)$ & $(0,0)$ & $(0,0)$ & $(0, b I)$ & $(0, b I)$ & $(0,0)$ & $(0, b I)$ & $(0, b I)$ \\
$(0, I)$ & $(0,0)$ & $(0, a I)$ & $(0, b I)$ & $(0, I)$ & $(0, a I)$ & $(0, b I)$ & $(0, I)$ \\
\hline
\end{tabular}

Then $\left(X(I),-{ }_{N}, \cdot\right)$ is a neutrosophic subtraction semi-group.

Proposition 3.14. Every neutrosophic subtraction semi-group is a subtraction semi-group.

Proof:

1. That $\left(X(I),-_{N}\right)$ is a subtraction algebra follows from Proposition 3.4 .

2. We need to show that $(X(I), \cdot)$ is a semi-group.

(a) Let $(a, b I),(c, d I) \in X(I)$ with $a, b, c, d \in X$, then $(a, b I) \cdot(c, d I)=(a c,(a d+b c+b d) I) \in X(I)$.

(b) $(a, b I),(c, d I),(e, f I) \in X(I)$ with $a, b, c, d, e, f \in X$,

We want to show that $((a, b I) \cdot(c, d I)) \cdot(e, f I)=(a, b I) \cdot((c, d I) \cdot(e, f I))$

$((a, b I) \cdot(c, d I)) \cdot(e, f I)=(a c,(a d+b c+b d) I) \cdot(e, f I)$

$$
\begin{aligned}
& =(a c e,(a c f+a d e+b c e+b d e+a d f+b c f+b d f) I) \\
& =(a, b I) \cdot(c e,(c f+d e+d f) I) \\
& =(a, b I) \cdot((c, d I) \cdot(e, f I)) .
\end{aligned}
$$

3. Let $(a, b I),(c, d I),(e, f I) \in X(I)$ then

(a) We shall show that $(a, b I) \cdot\left((c, d I)-{ }_{N}(e, f I)\right)=(a, b I) \cdot(c, d I)-{ }_{N}(a, b I) \cdot(e, f I)$

$(a, b I) \cdot\left((c, d I)-{ }_{N}(e, f I)\right)=(a, b I) \cdot(c-e,(d-f) I)$

$$
\begin{aligned}
& =(a(c-e),(a(d-f)+b(c-e)+b(d-f)) I) \\
& =(a c-a e,((a d-a f)+(b c-b e)+(b d-b f)) I) \\
& =(a c-a e,((a d+b c+b d)-(a f+b e+b f)) I) \\
& =(a c,(a d+b c+b d) I)-N(a e,(a f+b e+b f) I) \\
& =(a, b I) \cdot(c, d I)-{ }_{N}(a, b I) \cdot(e, f I) .
\end{aligned}
$$


(b) We shall show that $\left((c, d I)-{ }_{N}(e, f I)\right) \cdot(a, b I)=(c, d I) \cdot(a, b I)-{ }_{N}(e, f I) \cdot(a, b I)$

$$
\begin{aligned}
\left((c, d I)-{ }_{N}(e, f I)\right) \cdot(a, b I) & =((c-e,(d-f) I) \cdot(a, b I) \\
& =((c-e) a,((c-e) b+(d-f) a+(d-f) b) I) \\
& =((c a-e a),((c b-e b)+(d a-f a)+(d b-f b)) I) \\
& =((c a-e a),((c b+d a+d b)-(e b+f a+f b)) I) \\
& =(c a,(c b+d a+d b) I)-(e a,(e b+f a+f b) I) \\
& =(c, d I) \cdot(a, b I)-{ }_{N}(e, f I) \cdot(a, b I) .
\end{aligned}
$$

Definition 3.15. Let $X_{1}(I)$ and $X_{2}(I)$ be two neutrosophic subtraction semigroups. The direct product of $X_{1}(I)$ and $X_{2}(I)$ denoted by $X_{1}(I) \times X_{2}(I)$ is defined by $X_{1}(I) \times X_{2}(I)=\left\{\left(\left(a_{1}, b_{1} I\right),\left(a_{2}, b_{2} I\right)\right):\left(a_{1}, b_{1} I\right) \in X_{1}(I),\left(a_{2}, b_{2} I\right) \in X_{2}(I)\right\}$.

Proposition 3.16. Let $\left(X_{1}(I),-N_{1}, \cdot_{1}\right)$ and $\left(X_{2}(I),-N_{2}, \cdot 2\right)$ be two neutrosophic subtraction semigroup then $\left(X_{1}(I) \times X_{2}(I),-{ }_{N}, \cdot\right)$ is a neutrosophic subtraction semigroup.

Proposition 3.17. Let $\left(X(I),-_{N}, \cdot\right)$ be a neutrosophic subtraction semigroup and let $(A,-, \cdot)$ be a classical subtraction semigroup then $\left(X(I) \times A,-{ }_{N}, \cdot\right)$ is a neutrosophic subtraction semigroup.

Proposition 3.18. Let $\left(X(I),-{ }_{N}, \cdot\right)$ be a neutrosophic subtraction semigroup.

And let $(x, y I) \wedge(u, v I)=(x, y I)-{ }_{N}\left((x, y I)-{ }_{N}(u, v I)\right)$ for all $(x, y I),(u, v I),(s, t I) \in X(I)$.

Then the following are true.

1. $(x, y I)(0,0)$ and $(0,0)(x, y I)=(0,0)$

2. $(x, y I) \leq(u, v I)$ implies $(s, t I)(x, y I) \leq(s, t I)(u, v I)$ and $(x, y I)(s, t I) \leq(u, v I)(s, t I)$.

3. $(x, y I)((u, v I) \wedge(s, t I))=(x, y I)(u, v I) \wedge(x, y I)(s, t I)$ and $((x, y I) \wedge(u, v I))(s, t I)=(x, y I)(s, t I) \wedge(u, v I)(s, t I)$

4. $((x, y I) \wedge(u, v I))-{ }_{N}((x, y I) \wedge(s, t I)) \leq(x, y I) \wedge\left((u, v I)-{ }_{N}(s, t I)\right)$

Proof :

1. Let $(x, y I),(0,0) \in X(I)$ then

$$
\begin{aligned}
(x, y I)(0,0) & =(x, y I)\left((0,0)-{ }_{N}(0,0)\right) \\
& =(x, y I)(0,0)-{ }_{N}(x, y I)(0,0) \\
& =(0,0) . \\
\text { and } & \\
(0,0)(x, y I) & =\left((0,0)-{ }_{N}(0,0)\right)(x, y I) \\
& =(0,0)(x, y I)-{ }_{N}(0,0)(x, y I) \\
& =(0,0) .
\end{aligned}
$$

2. Let $(x, y I),(u, v I) \in X(I)$ and let $(x, y I) \leq(u, v I)$. Then we have

$$
(x, y I)-{ }_{N}(u, v I)=(x-u,(y-v) I)=(0,0) \Longrightarrow x-u=0 \quad \text { and } \quad y-v=0 \text {. }
$$

Now consider

$$
\begin{aligned}
& (s, t I)(x, y I)-{ }_{N}(s, t I)(u, v I)=(s x,(s y+t x+t y) I)-{ }_{N}(s u,(s v+t u+t v) I) \\
& =s x-s u,((s y-s v)+(t x-t u)+(t y-t v)) I \\
& =s(x-u),((s(y-v)+t(x-u)+t(y-v)) I \\
& =s(0),(s(0)+t(0)+t(0)) I \\
& \Longrightarrow(s, t I)(x, y I) \leq(s, t I)(u, v I) \text {. } \\
& =(0,0) \text {. }
\end{aligned}
$$

Following similar approach we can also prove that $(x, y I)(s, t I) \leq(u, v I)(s, t I)$.

3. Let $(x, y I),(u, v I),(s, t I) \in X(I)$ then

$$
\begin{aligned}
(x, y I)((u, v I) \wedge(s, t I)) & =(x, y I)\left((u, v I)-_{N}\left((u, v I)-{ }_{N}(s, t I)\right)\right) \\
& =(x, y I)(u, v I)-{ }_{N}(x, y I)\left((u, v I)-{ }_{N}(s, t I)\right) \\
& =(x, y I)(u, v I)-{ }_{N}\left((x, y I)(u, v I)-{ }_{N}(x, y I)(s, t I)\right) \\
& =(x, y I)(u, v I) \wedge(x, y I)(s, t I) .
\end{aligned}
$$

Following similar procedure we can show that

$((x, y I) \wedge(u, v I))(s, t I)=(x, y I)(s, t I) \wedge(u, v I)(s, t I)$. 
4. Let $(x, y I),(u, v I),(s, t I) \in X(I)$

$$
\begin{aligned}
&((x, y I) \wedge(u, v I))-{ }_{N}((x, y I) \wedge(s, t I))= {\left[(x, y I)-{ }_{N}\left((x, y I)-{ }_{N}(u, v I)\right)\right] } \\
&-{ }_{N}[(x, y I)-N((x, y I)-N(s, t I))] \\
&= {[x-(x-u),(y-(y-v)) I] } \\
&-N[x-(x-s),(y-(y-t)) I] \\
& \leq {[(x-s),(y-t) I]-N[(x-u),(y-v)) I] } \\
& \leq((x, y I)-N(s, t I))-{ }_{N}\left((x, y I)-{ }_{N}(u, v I)\right) \\
& \leq(u, v I)-{ }_{N}(s, t I) \\
& \leq(x, y I) \wedge(u, v I)) \\
& \text { Also, we have that } \quad \cdots \cdots(1) \\
&((x, y I) \wedge(u, v I))-{ }_{N}((x, y I) \wedge(s, t I)) \\
& \leq(x, y I) \quad \cdots \cdots(2)
\end{aligned}
$$

Combining 1 and 2 we have $((x, y I) \wedge(u, v I))-{ }_{N}((x, y I) \wedge(s, t I)) \leq(x, y I) \wedge\left((u, v I)-{ }_{N}(s, t I)\right)$.

Proposition 3.19. Let $X(I)$ be a neutrosophic subtraction semigroup. Then the following hold:

1. $(x, y I) \wedge\left((x, y I)-{ }_{N}(a, b I)\right)=(x, y I)-{ }_{N}(a, b I)$

2. $(x, y I) \wedge\left((a, b I)-{ }_{N}(x, y I)\right)=(0,0 I)$

3. $\left((x, y I)-{ }_{N}(a, b I)\right) \wedge\left((a, b I)-{ }_{N}(x, y I)\right)=(0,0 I)$

4. $\left((x, y I)-{ }_{N}(c, d I)\right) \wedge\left((a, b I)-{ }_{N}(c, d I)\right)=\left((x, y I)-{ }_{N}(c, d I)\right) \wedge(a, b I)$

Proof:

1. For any $(x, y I),(a, b I) \in X(I)$ we have

$$
\begin{aligned}
(x, y I) \wedge\left((x, y I)-{ }_{N}(a, b I)\right) & =(x, y I)-{ }_{N}\left((x, y I)-{ }_{N}\left((x, y I)-{ }_{N}(a, b I)\right)\right) \\
& =(x, y I)-{ }_{N}(a, b I) . \text { From } 6 \text { of 3.6. }
\end{aligned}
$$

2. For any $(x, y I),(a, b I) \in X(I)$ we have

$$
\begin{aligned}
(x, y I) \wedge\left((a, b I)-{ }_{N}(x, y I)\right) & =(x, y I)-{ }_{N}\left((x, y I)-{ }_{N}\left((a, b I)-{ }_{N}(x, y I)\right)\right) \\
& =(x, y I)-{ }_{N}\left((x, y I)-{ }_{N}((a-x,(b-y) I))\right. \\
& \left.=(x, y I)-{ }_{N}(x-(a-x), y-(b-y)) I\right) \\
& =(x, y I)-{ }_{N}(x, y I) \quad \text { from } 1 \text { of } 2.1 \\
& =(0,0) .
\end{aligned}
$$

3. For any $(x, y I),(a, b I) \in X(I)$ we have

$$
\begin{aligned}
\left((x, y I)-{ }_{N}(a, b I)\right) \wedge\left((a, b I)-{ }_{N}(x, y I)\right)= & \left((x, y I)-{ }_{N}(a, b I)\right) \\
& -{ }_{N}\left(\left((x, y I)-{ }_{N}(a, b I)\right)-{ }_{N}\left((a, b I)-{ }_{N}(x, y I)\right)\right) \\
= & \left((x, y I)-{ }_{N}(a, b I)\right)-{ }_{N}\left((x, y I)-{ }_{N}(a, b I)\right) \\
& \text { from 5 of 3.6 } \\
= & (0,0 I) .
\end{aligned}
$$

4. For any $(x, y I),(a, b I),(c, d I) \in X(I)$ we have

$$
\begin{aligned}
\left((x, y I)-{ }_{N}(c, d I)\right) \wedge\left((a, b I)-{ }_{N}(c, d I)\right)= & \left((x, y I)-{ }_{N}(c, d I)\right) \\
& -{ }_{N}\left(\left((x, y I)-{ }_{N}(c, d I)\right)-{ }_{N}\left((a, b I)-{ }_{N}(c, d I)\right)\right) \\
= & \left((x, y I)-{ }_{N}(c, d I)\right)-{ }_{N}\left(\left((x, y I)-{ }_{N}(a, b I)\right)-{ }_{N}(c, d I)\right) \\
= & \left((x, y I)-{ }_{N}(c, d I)\right)-N_{N}\left(\left((x, y I)-{ }_{N}(c, d I)\right)-{ }_{N}(a, b I)\right) \\
= & \left((x, y I)-{ }_{N}(c, d I)\right) \wedge(a, b I) .
\end{aligned}
$$

Definition 3.20. Let $\left(X(I),{ }_{N}, \cdot\right)$ be a neutrosophic subtraction semigroup and let $S(I)$ be a nonempty subset of $X(I) . S(I)$ is called a neutrosophic subtraction subsemigroup of $X(I)$ if $\left(S(I),{ }_{N}, \cdot\right)$ is itself a neutrosophic subtraction semigroup. It is essential that $S(I)$ contains a proper subset which is a subtraction semigroup. Otherwise, $S(I)$ will be called a pseudo neutrosophic subtraction subsemigroup of $X(I)$.

Example 3.21. Let $X(I)=\{(0,0),(a, 0),(b, 0),(1,0),(0, a I),(0, b I),(0, I)\}$ be a neutrosophic subtraction semigroup. The set $S(I)=\{(0,0),(a, 0),(b, 0),(1,0),(0, a I),(0, b I)\}$ is a neutrosophic subtraction subsemigroup.

Example 3.22. Let $X(I)=\{(0,0),(a, 0),(b, 0),(1,0),(0, a I),(0, b I),(0, I)\}$ be a neutrosophic subtraction semigroup. The set $S(I)=\{(0,0),(0, a I),(0, b I),(0, I)\}$ is a pseudo neutrosophic subtraction subsemigroup. 
Definition 3.23. Let $\left(X(I),{ }_{N}, \cdot\right)$ be a neutrosophic subtraction semigroup. A nonempty subset $A(I)$ of $X(I)$ is called

1. a left neutrosophic ideal if $A(I)$ is a neutrosophic subalgebra of $\left(X(I),{ }_{N}\right)$ and $X(I) A(I) \subseteq A(I)$

2. a right neutrosophic ideal if $A(I)$ is a neutrosophic subalgebra of $\left(X(I),{ }_{N}\right)$ and $A(I) X(I) \subseteq A(I)$

3. a neutrosophic ideal if $A(I)$ is both a left neutrosophic and right neutrosophic ideal.

Example 3.24. Let $X(I)$ be a neutrososphic subtraction semigroup and $(a, b I) \in X(I)$. Then

$$
X(I)(a, b I)=\{(x, y I)(a, b I):(x, y I) \in X(I)\}
$$

is a left neutrososphic ideal of $X(I)$.

Proposition 3.25. Let $X(I)$ be a neutrosophic subtraction semigroup. If $A(I)$ is any ideal of $X(I)$. Then $\bigcap_{i=1}^{n} A_{i}(I)$ is a neutrosophic ideal of $X(I)$. Where $\left\{A_{i}(I)\right\}_{i=1}^{n}$ is a family of neutrosophic ideals of $X(I)$.

Proof: Same as in classical sense.

Proposition 3.26. Let $A(I)$ be a neutrosophic ideal of a neutrosophic subtraction semigroup $X(I)$. If $(x, y I) \leq(u, v I)$ and $(u, v I) \in A(I)$, then $(x, y I) \in A(I)$

Proof: Since $(x, y I) \leq(u, v I)$ then $(x, y I)-{ }_{N}(u, v I)=(x-u,(y-v) I)=(0,0)$

$\Longrightarrow x-u=0$ and $y-v=0$

Now consider

$(u, v I)=(u, v I)-{ }_{N}\left((u, v I)-{ }_{N}(x, y I)\right) \quad$ from 6 of 3.6

$=(x, y I)-{ }_{N}\left((x, y I)-{ }_{N}(u, v I)\right)$

$=(x, y I)-{ }_{N}((x-u),(y-v) I)$

$=(x, y I)-{ }_{N}(0,0)$

$=(x, y) \in A(I)$.

Proposition 3.27. Let $A(I)$ be neutrosophic ideal of a neutrosophic subtraction semigroup $X(I)$. For any element $(a, b I) \in X(I)$ the set

$$
A(I)_{(a, b I)}=\{(u, v I) \in X(I):(a, b I) \wedge(u, v I) \in A(I)\}
$$

is a neutrosophic ideal of $X(I)$.

Proof:

1. $A(I)_{(a, b I)} \neq \emptyset$, since

$$
\begin{aligned}
(a, b I) \wedge(0,0) & =(a, b I)-{ }_{N}\left((a, b I)-{ }_{N}(0,0)\right) \\
& =(a, b I)-{ }_{N}(a, b I) \\
& =(0,0) \in A(I)
\end{aligned}
$$

Then we have that $(0,0) \in A(I)_{(a, b I)}$.

2. Let $x=(u, v I), y=(p, q I) \in X(I)$. and $z=(r, s I) \in A(I)_{(a, b I)}$

then $(a, b I) \wedge(r, s I) \in A(I)$

Then we shall show that $x z-{ }_{N} x\left(y-{ }_{N} z\right) \in A(I)_{(a, b I)}$.

Now consider

$$
\begin{aligned}
(a, b I) \wedge\left(x z-{ }_{N} x\left(y-{ }_{N} z\right)\right)= & (a, b I) \wedge((u, v I)(r, s I))-_{N}(a, b I) \wedge(u, v I)\left((p, q I)-_{N}(r, s I)\right) \\
& \operatorname{By} 3 \text { of } 3.19 \\
= & (a, b I) \wedge((u, v I)(r, s I))-_{N}(a, b I) \wedge\left((u, v I)(p, q I)-{ }_{N}(u, v I)(r, s I)\right) \\
= & (a, b I) \wedge((u, v I)(r, s I))-{ }_{N} \\
& {\left[(a, b I) \wedge((u, v I)(p, q I))-{ }_{N}(a, b I) \wedge((u, v I)(r, s I))\right] } \\
& \text { By } 1 \text { of } 2.1 \\
= & (a, b I) \wedge((u, v I)(r, s I)) \in A(I)
\end{aligned}
$$

$\Longrightarrow(a, b I) \wedge\left(x z-{ }_{N} x\left(y-{ }_{N} z\right)\right) \in A(I)$ and hence $\left(x z-{ }_{N} x\left(y-{ }_{N} z\right)\right) \in A(I)_{(a, b I)}$.

3. Let $x=(u, v I) \in X(I)$ we have that

$$
\begin{aligned}
A(I)_{(a, b I)} X(I)=A(I)_{(a, b I)}(u, v I) & =\{(m, n I) \mid(a, b I) \wedge(m, n I) \in A(I)\}(u, v I) \\
& =\{(m, n I)(u, v I) \mid(a, b I) \wedge(m, n I)(u, v I) \in A(I)\} . \\
& \subseteq A(I)_{(a, b I)} .
\end{aligned}
$$


Hence $A(I)_{(a, b I)}$ is an ideal of $X(I)$.

Proposition 3.28. Let $X(I)$ be a neutrosophic subtraction semigroup. If $A(I)$ and $B(I)$ are any two neutrosophic ideals of $X(I)$ then

1. $A(I)-{ }_{N} B(I)=\left\{a-{ }_{N} b: a \in A(I), b \in B(I)\right\}$ is a neutrosophic ideal of $X(I)$

2. $A(I) B(I)=\left\{\sum_{i=1}^{n} a_{i} b_{i}: a_{i} \in A(I), b_{i} \in B(I)\right\}$ is a neutrosophic ideal of $X(I)$

Proof:

1. To see that $A(I)-{ }_{N} B(I)$ is a neutrosophic ideal of $X(I)$.

Let $x=(u, v I), y=(p, q I) \in X(I)$, and $z=a-{ }_{N} b \in A(I)-{ }_{N} B(I)$. Where $a=(m, n I) \in A(I)$ and $b=(r, s I) \in B(I)$

Then

$$
\begin{aligned}
x z-{ }_{N} x\left(y-{ }_{N} z\right)= & x\left(a-{ }_{N} b\right)-{ }_{N} x\left(y-{ }_{N}\left(a-{ }_{N} b\right)\right) \\
= & (u, v I)\left((m, n I)-{ }_{N}(r, s I)\right)-{ }_{N}(u, v I)\left[(p, q I)-{ }_{N}\left((m, n I)-{ }_{N}(r, s I)\right)\right] \\
= & (u, v I)\left((m, n I)-{ }_{N}(r, s I)\right)-{ }_{N}\left[(u, v I)(p, q I)-{ }_{N}(u, v I)\left((m, n I)-{ }_{N}(r, s I)\right)\right] \\
= & {\left[(u, v I)(m, n I)-{ }_{N}(u, v I)(r, s I)\right] } \\
& -{ }_{N}\left[(u, v I)(p, q I)-{ }_{N}\left((u, v I)(m, n I)-{ }_{N}(u, v I)(r, s I)\right)\right] \\
= & {\left[(u m,(u n+v m+v n) I)-{ }_{N}(u r,(u s+v r+v s) I) I\right] } \\
& -{ }_{N}\left[(u p,(u q+v p+v q) I)-{ }_{N}\left[(u m,(u n+v m+v n) I)-{ }_{N}(u r,(u s+v r+v s) I)\right]\right] \\
= & {[(u m-u r),((u n-u s)+(v m-v r)+(v n-v s)) I] } \\
& -{ }_{N}\left[(u p,(u q+v p+v q) I)-{ }_{N}[(u m-u r),((u n-u s)+(v m-v r)+(v n-v s)) I]\right] \\
= & {[(u m-u r),((u n-u s)+(v m-v r)+(v n-v s)) I] } \\
& -{ }_{N}[(u p-(u m-u r)),((u q+v p+v q)-((u n-u s)+(v m-v r)+(v n-v s))) I] \\
= & (u m-u r)-[u p-(u m-u r)],((u n-u s)+(v m-v r)+(v n-v s)) I \\
& -[(u q+v p+v q)-((u n-u s)+(v m-v r)+(v n-v s))] I \\
= & (u m-u r)-[u p-(u m-u r)],((u n-u s)-[u q-(u n-u s)] \\
& +(v m-v r)-[v p-(v m-v r)]+(v n-v s)-[v q-(v n-v s)]) I \\
= & (u m-u r),[(u n-u s)+(v m-v r)+(v n-v s)] \quad \text { By } 1 \text { of } 2.1] \\
= & {[u m,(u n+v m+v n) I]-{ }_{N}[u r,(u s+v r+v s) I] } \\
= & (u, v I)(m, n I)-{ }_{N}(u, v I)(r, s I) \\
= & (u, v I)\left[(m, n I)-{ }_{N}(r, s I)\right] \\
= & x\left(a-{ }_{N} b\right) \\
= & x z \in A(I)-{ }_{N} B(I) . \\
\text { And } & =\left\{z x: z \in A(I)-{ }_{N} B(I), x \in X(I)\right\} \\
\left(A(I)-{ }_{N} B(I)\right) X(I) & \subseteq(I)-{ }_{N} B(I) .
\end{aligned}
$$

So, we say that $A(I)-{ }_{N} B(I)$ is a neutrosophic ideal.

2. To see that $A(I) B(I)$ is a neutrosophic ideal.

- $A(I) B(I) \neq \emptyset$ since $A(I)$ and $B(I)$ are both neutrosophic ideals of $X(I)$. So $(0,0) \in A(I) B(I)$

- Let $x=(u, v I), y=(p, q I) \in X(I)$, and $z=\sum_{i=1}^{n} a_{i} b_{i} \in A(I) B(I)$. Where $a_{i}=\left(m_{i}, n_{i} I\right) \in$ $A(I)$ and $b_{i}=\left(r_{i}, s_{i} I\right) \in B(I)$

Then 


$$
\begin{aligned}
x z-{ }_{N} x\left(y-{ }_{N} z\right)= & x \sum_{i=1}^{n} a_{i} b_{i}-N x\left(y-N \sum_{i=1}^{n} a_{i} b_{i}\right) \\
= & (u, v I) \sum_{i=1}^{n}\left(m_{i}, n_{i} I\right)\left(r_{i}, s_{i} I\right)-N(u, v I)\left[(p, q I)-{ }_{N} \sum_{i=1}^{n}\left(m_{i}-n_{i} I\right)\left(r_{i}, s_{i} I\right)\right] \\
= & (u, v I) \sum_{i=1}^{n}\left(m_{i} r_{i},\left(m_{i} s_{i}+n_{i} r_{i}+n_{i} s_{i}\right) I\right) \\
& -{ }_{N}\left[(u, v I)(p, q I)-N(u, v I) \sum_{i=1}^{n}\left(m_{i} r_{i},\left(m_{i} s_{i}+n_{i} r_{i}+n_{i} s_{i}\right) I\right)\right] \\
= & \sum_{i=1}^{n}\left[(u, v I)\left(m_{i} r_{i},\left(m_{i} s_{i}+n_{i} r_{i}+n_{i} s_{i}\right) I\right)\right. \\
& \left.-{ }_{N}\left[u p,(u q+v p+v q) I-N(u, v I)\left(m_{i} r_{i},\left(m_{i} s_{i}+n_{i} r_{i}+n_{i} s_{i}\right) I\right)\right]\right] \\
= & \sum_{i=1}^{n}\left[\left(u m_{i} r_{i},\left(u m_{i} s_{i}+u n_{i} r_{i}+u n_{i} s_{i}+v m_{i} r_{i}+v m_{i} s_{i}+v n_{i} r_{i}+v n_{i} s_{i}\right) I\right)\right. \\
& -{ }_{N}\left[u p,(u q+v p+v q) I-{ }_{N}\left(u m_{i} r_{i},\left(u m_{i} s_{i}+u n_{i} r_{i}+u n_{i} s_{i}+v m_{i} r_{i}+v m_{i} s_{i}\right.\right.\right. \\
& \left.\left.\left.\left.+v n_{i} r_{i}+v n_{i} s_{i}\right) I\right)\right]\right] \\
= & \left(u m_{i} s_{i}+u n_{i} r_{i}+u n_{i} s_{i}+v m_{i} r_{i}+v m_{i} s_{i}+v n_{i} r_{i}+v n_{i} s_{i}\right) \\
= & (u q+v p+v q) \\
\text { Take } \alpha & \\
\text { and } \beta & \sum_{i=1}^{n}\left[\left(u m_{i} r_{i}, \alpha I\right)-{ }_{N}\left[(u p, \beta I)-{ }_{N}\left(u m_{i} r_{i}, \alpha I\right)\right]\right] \\
\text { Then } x z-{ }_{N} x\left(y-{ }_{N} z=\right. & \sum_{i=1}^{n}\left[u m_{i} r_{i}-\left[u p-\left(u m_{i} r_{i}\right)\right],(\alpha-[\beta-\alpha]) I\right] \\
= & \sum_{i=1}^{n}\left[u m_{i} r_{i}, \alpha I\right] \\
= & \sum_{i=1}^{n}\left[u m_{i} r_{i},\left(u m_{i} s_{i}+u n_{i} r_{i}+u n_{i} s_{i}+v m_{i} r_{i}+v m_{i} s_{i}+v n_{i} r_{i}+v n_{i} s_{i}\right) I\right] \\
= & \sum_{i=1}^{n}(u, v I)\left[m_{i} r_{i},\left(m_{i} s_{i}+n_{i} r_{i}+n_{i} s_{i}\right) I\right] \\
= & (u, v I) \sum_{i=1}^{n}\left(m_{i} r_{i},\left(m_{i} s_{i}+n_{i} r_{i}+n_{i} s_{i}\right) I\right) \\
= & (u, v I) \sum_{i=1}^{n}\left(m_{i}, n_{i} I\right)\left(r_{i}, s_{i} I\right) \\
= & (u, v I) \sum_{i=1}^{n} a_{i} b_{i} \\
= & x z .
\end{aligned}
$$

- For $z \in A(I) B(I)$ and $x \in X(I)$ we have that

$$
\begin{aligned}
z x & =\left(\sum_{i=1}^{n} a_{i} b_{i}\right)(u, v I) \\
& =\sum_{i=1}^{n}\left(m_{i}, n_{i} I\right)\left(r_{i}, s_{i} I\right)(u, v I) \\
& =\sum_{i=1}^{n}\left(m_{i} r_{i},\left(m_{i} s_{i}+n_{i} r_{i}+n_{i} s_{i}\right) I\right)(u, v I) \\
& =\sum_{i=1}^{n}\left(m_{i} r_{i} u,\left(m_{i} r_{i} v+m_{i} s_{i} u+n_{i} r_{i} u+n_{i} s_{i} u+m_{i} s_{i} v+n_{i} r_{i} v+n_{i} s_{i} v\right) I\right) \in A(I) B(I)
\end{aligned}
$$

Then we can say that $A(I) B(I) x \subseteq A(I) B(I)$, Hence $A(I) B(I)$ is a neutrosophic ideal.

Definition 3.29. Let $(S,-, \cdot)$ be any near subtraction semigroup. The triple $\left(S(I),{ }_{N}, \cdot\right)$ is called a neutrosophic near subtraction semigroup. If $(a, b I)$ and $(c, d I)$ are any two elements of $S(I)$ with $a, b, c, d \in S$, we define

$$
(a, b I)-{ }_{N}(c, d I)=(a-c,(b-d) I)
$$

and

$$
(a, b I) \cdot(c, d I)=(a c,(a d+b c+b d) I)
$$

Example 3.30. Let $\left(S(I),{ }_{N}\right)$ be a neutrosophic subtraction algebra. Then the neutrosophic set $M^{S(I)}$ of all mappings of $S(I)$ into $S(I)$ is a neutrososphic near subtraction semigroup under ${ }_{N}$ (Defined point-wisely) and composition of mappings. I.e For all $\phi, \psi \in M^{S(I)}$ we define

$$
\left(\phi-{ }_{N} \psi\right)(a, b I)=\phi(a, b I)-{ }_{N} \psi(a, b I)
$$

and

$$
(\phi o \psi)(a, b I)=\phi o(\psi(a, b I))
$$

$M^{S(I)}$ is not a neutrosophic subtraction semigroup, because if $\phi_{(0, I)}: M^{S(I)} \longrightarrow M^{S(I)}$ is given by $\phi_{(0, I)}(\alpha)=(0, I)$ for all $\alpha \in M^{S(I)}\left(\phi_{(0, I)}\right.$ is a constant map). Then for any $\rho, \beta \in M^{S(I)}$, $\phi_{(0, I)}=\phi_{(0, I)} o\left(\rho-{ }_{N} \beta\right) \neq \phi_{(0, I)} o \rho-{ }_{N} \phi_{(0, I)} o \beta=(0,0)$

Proposition 3.31. Let $\left(S(I),{ }_{N}, \cdot\right)$ be a neutrosophic near subtraction semigroup. Then $S(I)$ is a near subtraction semigroup.

Proof: Follows similar approach as the proof of Proposition 3.14.

Example 3.32. Let $X(I)=\{(0,0),(a, 0),(b, 0),(1,0),(0, a I),(0, b I),(0, I)\}$. For all $(u, v I) \in X(I)$ with $u, v \in X$. If $u \cdot v=u$. Then $\left(S(I),{ }_{N}, \cdot\right)$ is a neutrosophic near subtraction semigroup.

Proposition 3.33. Let $X(I)$ be a neutrosophic left near subtraction semigroup. If $A(I)$ and $B(I)$ are any two neutrosophic ideals of $X(I)$ then $(A(I): B(I))=\{x \in X(I): x B(I) \subseteq A(I)\}$ is a neutrosophic left ideal of $X(I)$. 
Proof: Let $a=(m, n I), b=(r, s I) \in(A(I): B(I))$ and let $x=(u, v I) \in X(I)$.

To show that $A(I): B(I)$ is a neutrosophic left ideal of the neutrosophic near subtraction semigroup $X(I)$.

1. $A(I): B(I) \neq \emptyset$ since $A(I)$ and $B(I)$ are both neutrosophic ideals of $X(I)$.

2. We shall show that $a-{ }_{N} x b \in(A(I): B(I))$. To see this it suffices to show that $\left(a-{ }_{N} x b\right) B(I) \subseteq A(I)$. Let $k=(p, q I) \in B(I)$ be an arbitrary element. Then since $a=(m, n I), b=(r, s I) \in(A(I): B(I))$, we have $a k=(m p,(m q+n p+n q) I), b k=(r p,(r q+s p+s q) I) \in A(I)$.

Since $A(I)$ is a neutrosophic ideal of $X(I)$, we have

$x(b k)=(u, v I)(r p,(r q+s p+s q) I)=(u r p,(u r q+u s p+u s q+v r p+v r q+v s p+v s q) I) \in A(I)$

as well. Thus $\left(a-{ }_{N} x b\right) k=a k-{ }_{N} x(b k) \in A(I)$ for any $k \in B(I)$, and hence we obtain $\left(a-{ }_{N} x b\right) B(I) \subseteq A(I)$. By the definition of $(A(I): B(I))$, we have $a-{ }_{N} x b \in(A(I): B(I))$.

Hence $(A(I): B(I))$ is a neutrosophic left ideal of the neutrosophic near subtraction semigroup $X(I)$. In a right neutrosophic near subtraction semigroup $S(I), \quad(0,0)(u, v I)=(0,0)$ for all $(u, v I) \in S(I)$. But $(u, v I)(0,0 I)$ need not be equal to $(0,0)$, for $(u, v I) \in S(I)$. To this end, we have the following definition:

Definition 3.34. Let $\left(S(I),{ }_{N}, \cdot\right)$ be right neutrosophic near subtraction semigroup. $S(I)$ is said to be zerosymmetric if $S(I)=S_{(0,0)}(I)$, where

$$
S_{(0,0)}(I)=\{(u, v I) \in S(I):(u, v I)(0,0)=(0,0)\} .
$$

Example 3.35. Let $S(I)=\{(0,0),(1,0),(0, I)\}$, define ${ }^{\prime}-^{\prime}{ }_{N}$ and ${ }^{\prime} \cdot{ }^{\prime}$ as shown below:

Table 4: (i) Cayley table for the binary operation of " $-{ }_{N}$ " and (ii) Cayley table for the binary operation of ". ."

(i)

\begin{tabular}{llll}
\hline$-_{N}$ & $(0,0)$ & $(1,0)$ & $(0, I)$ \\
\hline$(0,0)$ & $(0,0)$ & $(0,0)$ & $(0,0)$ \\
$(1,0)$ & $(1,0)$ & $(0,0)$ & $(1,0)$ \\
$(0, I)$ & $(0, I)$ & $(0, I)$ & $(0,0)$ \\
\hline
\end{tabular}

(ii)

\begin{tabular}{llll}
\hline$\cdot$ & $(0,0)$ & $(1,0)$ & $(0, I)$ \\
\hline$(0,0)$ & $(0,0)$ & $(0,0)$ & $(0,0)$ \\
$(1,0)$ & $(0,0)$ & $(1,0)$ & $(0,0)$ \\
$(0, I)$ & $(0,0)$ & $(0,0)$ & $(0, I)$ \\
\hline
\end{tabular}

Then $\left(S(I),{ }_{N}, \cdot\right)$ is a zero symmetric right neutrosophic near subtraction semigroup.

Definition 3.36. Let $X(I)$ be a neutrosophic subtraction semigroup and let $A(I)$ be a neutrosophic ideal of $X(I)$. The set $X(I) / A(I)$ is defined by

$$
X(I) / A(I)=\{(u, v I)+A(I):(u, v I) \in X(I)\}
$$

For all $(u, v I)+A(I),(r, s I)+A(I) \in X(I) / A(I)$, we define subtraction and multiplication in $N(I) / A(I)$ as follows:

$$
\begin{gathered}
((u, v I)+A(I)) \ominus_{N}((r, s I)+A(I))=(u, v I)-{ }_{N}(r, s I)+A(I)=(u-r,(v-s) I)+A(I), \\
((u, v I)+A(I)) \odot((r, s I)+A(I))=(u, v I)(r, s I)+A(I)=(u r,(u s+v r+v s) I)+A(I)
\end{gathered}
$$

It can be shown that $\ominus_{N}$ and $\odot$ are well-defined on $N(I) / A(I)$ and the triple $\left(N(I) / A(I), \ominus_{N}, \odot\right)$ is a neutrosophic subtraction semigroup called neutrosophic quotient subtraction semigroup or neutrosophic factored subtraction semigroup. provided $X / A$ is a subtraction semigroup. 
Definition 3.37. Let $\left(A(I),-{ }_{N}, \cdot\right)$ and $\left(B(I),-{ }^{\prime}{ }_{N}, \cdot^{\prime}\right)$ be two neutrosophic subtraction semigroups. A mapping $\phi: A(I) \longrightarrow B(I)$ is called a neutrosophic subtraction semigroup homomorphism if the following conditions hold:

1. $\phi\left((a, b I)-_{N}(c, d I)\right)=\phi((a, b I))-^{\prime}{ }_{N} \phi((c, d I)), \forall(a, b I),(c, d I) \in A(I)$.

2. $\phi((a, b I) \cdot(c, d I))=\phi((a, b I)) \cdot^{\prime} \phi((c, d I)), \forall(a, b I),(c, d I) \in A(I)$.

3. $\phi((0, I))=(0, I)$

Epimorphism, monomorphism, isomorphism, endomorphism and automorphism of $\phi$ are similarly defined as in the classical cases.

Definition 3.38. Let $\phi: A(I) \longrightarrow B(I)$ be a neutrosophic subtraction semigroup homomorphism.

1. $\operatorname{Ker} \phi=\{(a, b I) \in A(I): \phi((a, b I))=(0,0)\}$.

2. $\operatorname{Im} \phi=\{\phi((a, b I)) \in B(I):(a, b I) \in A(I)\}$.

Example 3.39. Let $A(I)$ and $B(I)$ be two neutrosophic subtraction semigroup. Let $\phi: A(I) \times B(I) \longrightarrow A(I)$ be a mapping defined by $\phi(a, b)=a$ and let $\rho: A(I) \times B(I) \longrightarrow B(I)$ be a mapping defined by $\rho(a, b)=b$ for all $(a, b) \in A(I) \times B(I)$. Then $\phi$ and $\rho$ are neutrosophic subtraction semigroup homomorphisms.

Example 3.40. Let $X(I)=\{(0,0),(a, 0),(b, 0),(1,0),(0, a I),(0, b I),(0, I)\}$ be a neutrosophic subtraction semigroup. Let $\phi: X(I) \times X(I) \longrightarrow X(I)$ be a neutrosophic subtraction semigroup homomorphism defined by $\phi(p, q)=p$ for all $p, q \in X(I)$. Then

1. $\operatorname{Im} \phi=\{(0,0),(a, 0),(b, 0),(1,0),(0, a I),(0, b I),(0, I)\}$ which is a neutrosophic subtraction subsemigroup.

2. Also,

$$
\operatorname{Ker} \phi=\{((0,0),(0,0)),((0,0),(a, 0)),((0,0),(b, 0)),((0,0),(1,0)),((0,0),(0, a I)),((0,0),(1, b I)),((0,0),(0, I))\}
$$

which is just a subtraction subsemigroup, not a neutrosophic subtraction semigroup and equally not a neutrosophic ideal.

Example 3.40 leads to the following general result.

Proposition 3.41. Let $\phi: A(I) \longrightarrow B(I)$ be a neutrosophic subtraction semigroup homomorphism. Then

1. Im $\phi$ is a neutrosophic subtraction subsemigroup of $B(I)$.

2. Ker $\phi$ is a subtraction subsemigroup of $A(I)$.

3. Ker $\phi$ is not a neutrosophic subtraction subsemigroup of $A(I)$.

4. Ker $\phi$ is not a neutrosophic ideal of $A(I)$

Proof:

1. Same as in classical case.

2. By the definition of neutrosophic subtraction semigroup homomorphisms, we know that $\phi(I)=I$. Then, for any arbitrary $(x, y I) \in A(I)$ where $x, y \in A$, it follows that $(x, y I) \in \operatorname{Ker} \phi$ if and only if $y=0$ that is only elements of the form $(x, 0) \in A(I)$ can be in the kernel of $\phi$. Then $\operatorname{Ker} \phi$ is a subtraction subsemigroup of $A(I)$.

3. Since $\phi(I)=I$, it follows that $I \notin \operatorname{Ker} \phi$ and the result follows.

4. Since $\operatorname{Ker} \phi$ is a subtraction semigroup of $A(I)$ and not a neutrosophic subtraction semigroup of $A(I)$, it follows that $\operatorname{Ker} \phi$ cannot be a neutrosophic ideal of $A$.

\section{Conclusion}

In this paper, we have studied subtraction algebra and subtraction semigroup in the neutrosophic environment. Their basic properties have been extended and established in the neutrosophic environment. We hope to study and establish more advanced properties of subtraction algebra and subtractions semigroup in the neutrosophic environment in our future papers. 


\section{Acknowledgment}

The Authors which to thank the anonymous reviewers for their valuable and useful comments which have lead to the improvement of the paper.

\section{References}

[1] Agboola, A.A.A. and Davvaz, B., Introduction to Neutrosophic BCI/BCK-Algebras, International Journal of Mathematics and Mathematical Sciences. Article ID370267, 2015.

[2] Arshaduzzaman, M.D., A Characterization of a Group with Subtraction as a Binary Operation. International Organization of Scientific Research-Journal of Mathematics , vol. 10(1), pp. 53-55, 2014.

[3] Bakbak, D., Uluçay, V. and Şahin, M., Neutrosophic soft expert multiset and their application to multiple criteria decision making, Mathematics, vol. 7, pp. 50-65, 2019.

[4] Broumi, S., Bakali, A., Talea, M., Smarandache, F.,Singh, P. K., Uluçay, V., and Khan, M., Bipolar complex neutrosophic sets and its application in decision making problem. In Fuzzy Multi-criteria DecisionMaking Using Neutrosophic Sets. Springer, Cham. pp. 677-710, 2019.

[5] Jun,Y.B, Kim,H.S. and Roh, E.H, Ideal theory of subtraction algebras, Scientiae Mathematicae Japonicae Online, pp. 397-402, 2004.

[6] Jun, Y. B. and Kim, H.S. On ideals in subtraction algebras, Scientiae Mathematicae Japonicae, Online, pp. 1081-1086, 2006.

[7] Jun,Y.B and Kim,K.H., Prime and irreducible ideals in subtraction algebras, International Mathematical Forum, vol.3(10), pp. 457- 462, 2008.

[8] Kyung, H. K, Eun, H. R. and Yong, H. Y., A note on subtraction semigroup. Scientiae Mathematicae Japonicae Online, vol. 10, pp. 393-401, 2004.

[9] Lee, K. J., Jun, Y.B. and Kim, Y.H., Weak forms of subtraction algebras, Bulletin of the Korean Mathematical Society, vol. 45(3), pp. 437-444, 2008.

[10] Şahin, M. and Kargın A., Neutrosophic triplet groups based on set valued neutrosophic quadruple numbers, Neutrosophic Set and Systems, vol. 30, pp. 122 -131, 2019.

[11] Şahin M. and Kargın A., Neutrosophic Triplet v-Generalized Metric Space, Axioms- MDPI, vol.7, pp. 67-73, 2018.

[12] Şahin, M. and Kargın A., Single valued neutrosophic quadruple graphs, Asian Journal of Mathematics and Computer Research, vol. 26(4), pp. 243-250, 2019.

[13] Şahin M., Kargın A. and Çoban M. A., Fixed point theorem for neutrosophic triplet partial metric space, Symmetry- MDPI, vol.10(7), pp. 240-246, 2018.

[14] Schein, B.M., Difference semigroups, Communications in Algebra, vol. 20(8), pp. 2153-2169, 1992.

[15] Smarandache, F., A Unifying Field in Logics: Neutrosophic Logic, Neutrosophy, Neutrosophic Set, Neutrosophic Probability, American Research Press, Rehoboth, 2003.

[16] Smarandache, F., Introduction to Neutrosophic Statistics, Sitech and Education Publishing, Romania, 2014.

[17] Smarandache, F., Neutrosophic Probability, Set and Logic, Am Res Press, Rehoboth, USA, 1998.

[18] Ulucay, V., Kılıç, A., Şahin, M. and Deniz, H., A New Hybrid Distance-Based Similarity Measure for Refined Neutrosophic sets and its Application in Medical Diagnosis. MATEMATIKA: Malaysian Journal of Industrial and Applied Mathematics, vol. 35(1), pp 83-94, 2019.

[19] Uluçay, V., Kiliç, A.,Yildiz, I. and Sahin, M., A new approach for multi-attribute decision-making problems in bipolar neutrosophic sets. Neutrosophic Sets and Systems, vol. 23(1), pp.142-159, 2018. 
[20] Uluçay, V., Kılıç, A., Yıldız, İ. and Şahin, M., An Outranking Approach for MCDM-Problems with Neutrosophic Multi-Sets. Neutrosophic Sets and Systems, vol. 30, pp.214-24, 2019.

[21] Uluçay, V., and Şahin, M., Decision-Making Method based on Neutrosophic Soft Expert Graphs. In Neutrosophic Graph Theory and Algorithms. IGI Global, pp. 33-76, 2020.

[22] Ulucay, V., Şahin, M. and Olgun, N. Time-Neutrosophic Soft Expert Sets and Its Decision Making Problem. Matematika, vol. 34(2), pp. 246-260, 2018.

[23] Uluçay, V., Şahin, M., and Hassan, N., Generalized neutrosophic soft expert set for multiple-criteria decision-making. Symmetry, vol.10(10), pp. 437-455, 2018.

[24] Vasantha Kandasamy, W.B and Smarandsche,F., Neutrosophic Ring, Hexis Phoenix, Arizona 2006.

[25] Vansantha Kandasamy, W.B. and Smarandache,F., Some Neutrosophic Algebraic Structures and Neutrosophic N-Algebraic Structures, Hexis, Phoenix, Arizona, 2006.

[26] Yilmaz C. and Mehmet A.O., Some result on subtraction algebra. Hacettepe Journal of Mathematics and Statistics,vol. 38 (3), pp. 299-304, 2009.

[27] Zelinka, B., Subtraction semigroups. Mathematical Bohemica, vol. 120(4), pp. 445-447, 1995. 